\title{
Temporary Cross-Clamping of the Infrarenal Abdominal Aorta during Cesarean Hysterectomy to Control Operative Blood Loss
}

\author{
Mikio Hashiguchi, MD ${ }^{1}$ \\ ${ }^{1}$ Department of Maternal Fetal Medicine, Okinawa Chubu Hospital, \\ Okinawa, Japan \\ Address for correspondence Mikio Hashiguchi, MD, Department of \\ Maternal Fetal Medicine, Okinawa Chubu Hospital, 281 Miyazato \\ Uruma C, Okinawa 904-2293, Japan \\ Surg J (NY) 2021;7(suppl S1):S7-S10. \\ (e-mail: hashiguchi_mikio@hosp.pref.okinawa.jp).
}
Abstract
Keywords
- cross-clamping
- blood loss
- cesarean hysterectomy
- placenta accreta

Temporary cross-clamping of the infrarenal abdominal aorta to reduce bleeding during hysterectomy following cesarean section in patients with placenta previa-accreta is a relatively simple procedure; therefore, it can be an option when intravascular balloon catheter placement is impossible or encountered massive bleeding of the unexpected placenta accreta.
For reducing blood loss during hysterectomy for placenta previa-accreta, balloon catheter placement in the common iliac artery and abdominal aorta is widely performed in the recent years, and it was shown to be effective. However, there are only limited institutions capable of performing interventional radiology. Preparation for balloon catheter placement prior to laparotomy requires time, and placing the catheter within the blood vessels during surgery is difficult because sudden bleeding after laparotomy is a complex procedure. Furthermore, hypercoagulability occurs due to physiological changes caused by pregnancy, which increases the risk of thrombus formation by intravascular catheter placement. On the other hand, conventional artery ligation was performed via the internal iliac artery to reduce bleeding; however, in a pregnant uterus, collateral circulation develops, and patients with placenta previa-accreta have particularly abundant blood flow from external iliac artery system, making the effects of such ligation unreliable. Furthermore, when the lower portion of the uterus is swollen from the residual placenta, the visual field between the uterus and pelvic wall cannot be guaranteed, and the procedure is complicated because of the need for the left and right internal iliac arteries to be ligated, which therefore, results in the risk of venous injury having to be performed twice.

Infrarenal abdominal aortic clamping serves as a supportive measure of hemostasis treatment and is performed to reduce bleeding in artificial graft repair of abdominal aortic aneurysm and pelvic hemorrhage with pelvic fracture caused by trauma. It is a procedure performed by vascular surgeons in cooperation with expert general surgeons who are able to treat trauma patients.

Clamping of the infrarenal abdominal aorta to reduce bleeding during hysterectomy following cesarean section in patients with placenta previa-accreta is a relatively simple procedure with an anatomically easy approach; therefore, it can be an option when intravascular balloon catheter placement is impossible.

\section{Surgical Steps}

1. Extend the skin midline incision to the umbilicus. $\downarrow$ published online

September 9, 2021
DOI https://doi.org/ $10.1055 / \mathrm{s}-0041-1727253$. ISSN 2378-5128. (c) 2021. The Author(s).

This is an open access article published by Thieme under the terms of the Creative Commons Attribution-NonDerivative-NonCommercial-License, permitting copying and reproduction so long as the original work is given appropriate credit. Contents may not be used for commercial purposes, or adapted, remixed, transformed or built upon. (https://creativecommons.org/ licenses/by-nc-nd/4.0/)

Thieme Medical Publishers, Inc., 333 Seventh Avenue, 18th Floor, New York, NY 10001, USA 
2. Push the intestinal tract to the left and right to ensure the operative field.

$\downarrow$

3. Palpate the abdominal aorta and make an incision and open the retroperitoneum between the common iliac artery bifurcation and inferior mesenteric artery. $\downarrow$

4. Dissect the loose connective tissue surrounding the aorta and expose the aorta. $\downarrow$

5. Dissect along the arterial wall from the lower right side of the aorta using a dissecting forceps and penetrate into the lower left side. $\downarrow$

6. Place a vascular tape on the aorta and lift in a perpendicular manner. $\downarrow$

7. Clamp the aorta using a cross-clamp forceps. $\downarrow$

8. Following hysterectomy and upon completion of hemostasis treatment, gradually remove the cross-clamp forceps while observing blood pressure. $\downarrow$

9. Confirm the absence of ischemia caused by thrombus formation by palpating the pulse of the dorsal arteries of both the feet or by pulse oximeter of the large toe.

\section{Surgical Indications}

The surgical indications are:

- This method is used to reduce the blood loss during hysterectomy for patients with placenta previa-accreta.

- Cross clamping of the infrarenal abdominal aorta is performed in patients in whom balloon catheter placement in the common iliac artery or aorta could not be achieved preoperatively and in those with suspected extensive placental invasion into the bladder with abundant collateral circulation from the external iliac artery.

- In addition, this method is used to temporarily reduce the blood loss when massive, life-threatening bleeding occurs during pelvic surgery.

\section{Preoperative Preparations}

Request a vascular surgeon's support prior surgery. Place the patient in a lithotomy position, prepare adequate red blood cells, fresh frozen plasma, cryoprecipitate, and platelets should be available at surgery. Prepare heparin, palpate the dorsal arteries of both the feet, and mark the site of palpation.

\section{Surgical Instruments}

The surgical instruments required are: (1) aortic cross-clamp forceps and vascular tape; (2) self-retaining retractor designed to give adequate exposure.

\section{Surgical Technique}

Step 1: Securing adequate operative filed.

- Extend the skin midline incision to the umbilicus.

- Extend the skin midline incision to the umbilicus for manipulations above the abdominal aortic bifurcation.

- Push the intestinal tract to the left and right to ensure the operative field.

- The ascending colon, descending colon, and small intestine are pushed to the left, right, and upward to ensure the operative field. Another individual is needed as a second assistant to grasp the puerperal uterus. Using a retractor hook such as the type with two "wishbone" arms provides an option of attaching multiple retractors, thus freeing up the hands of the second assistant (-Fig. 1).

Step 2: Check the clamp site.

- Palpate the abdominal aorta and make an incision and open the retroperitoneum between the aortic bifurcation and inferior mesenteric artery.

- To cross-clamp position of the artery between the fourth lumbar vertebra from the aortic bifurcation, pick up the retroperitoneum directly above the pulsating aorta and open approximately $5 \mathrm{~cm}$ above and below.

Step 3: Exposing and freeing the wall of abdominal aorta.

- Dissect the loose connective tissue surrounding the aorta and expose the aorta.

- Dissect and release the connective tissue surrounding the aorta using dissecting forceps and an electrosurgical knife.

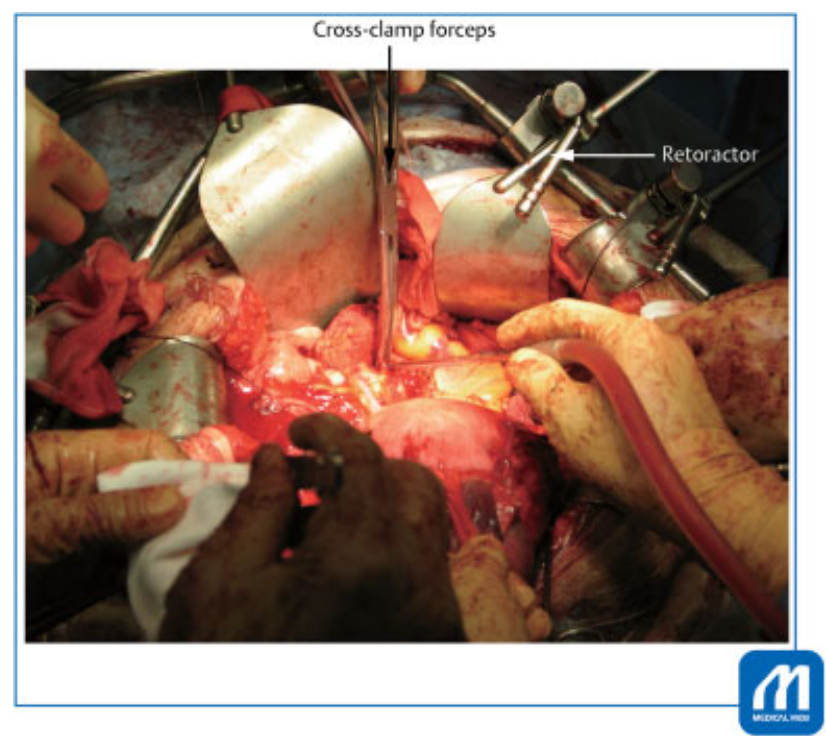

Fig. 1 Place a vascular tape on the aorta and lift in a perpendicular manner. To prevent clamping and injuring the vein with the crossclamp, slightly lift the artery using a vascular tape. Cross-clamp forceps; retoractor. (Reproduced with permission of Hashiguchi M. In: Hiramatsu Y, Konishi I, Sakuragi N, Takeda S, eds. Surgery for Pregnancy with Placenta Previa and Placenta Accrete: Careful Preparation and Critical Management. OGS NOW, No.9. (Japanese). Tokyo: Medical View; 2012:94-99. Copyright @ Medical View). 


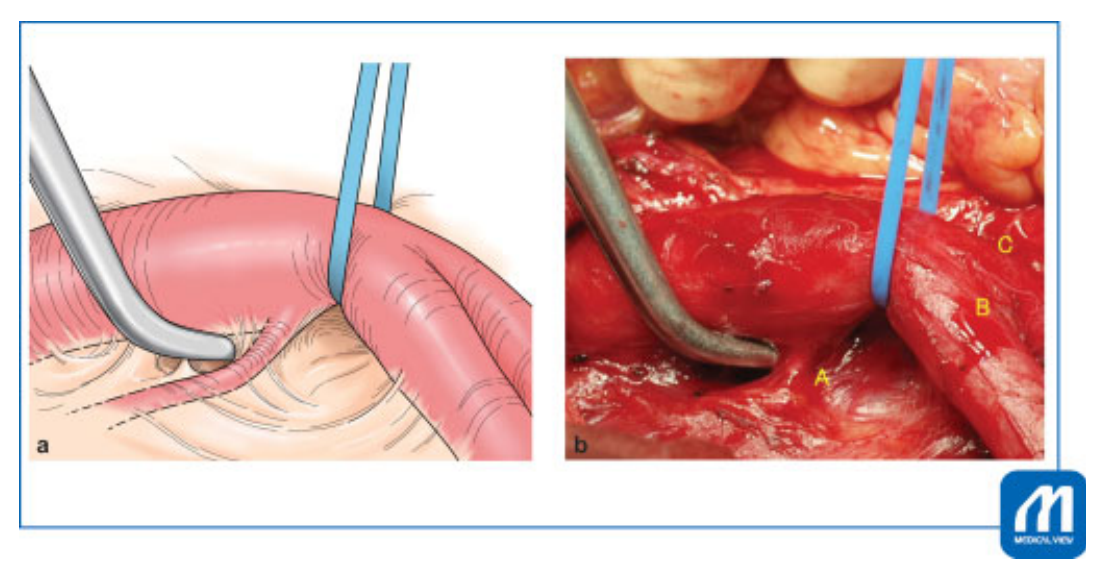

Fig. 2 Dissect along the arterial wall at a right angle from the lower right side of the aorta using dissecting forceps and penetrate into the lower left side. Caution should be exercised because the lumbar artery might be involved. (Reproduced with permission of Hashiguchi M. In: Hiramatsu Y, Konishi I, Sakuragi N, Takeda S, eds. Surgery for Pregnancy with Placenta Previa and Placenta Accrete: Careful Preparation and Critical Management. OGS NOW, No.9. (Japanese). Tokyo: Medical View; 2012:94-99. Copyright @ Medical View).

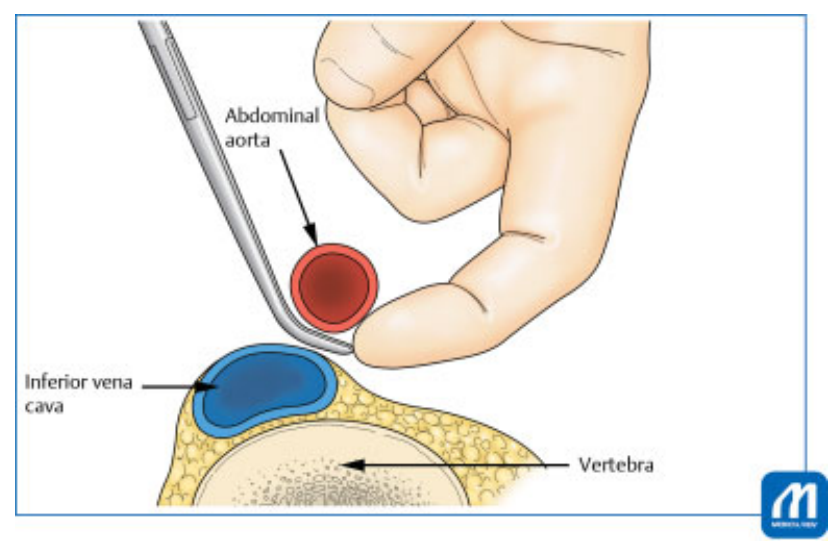

Fig. 3 Abdominal aorta, inferior vena cava, vertebra. Dissecting the arterial wall, place the index finger at the tip of the dissecting forceps, and verify the direction by which the forceps tip advances using the index finger and resistance of the forceps and the presence or absence of the lumber artery. (Reproduced with permission of Hashiguchi M. In: Hiramatsu Y, Konishi I, Sakuragi N, Takeda S, eds. Surgery for Pregnancy with Placenta Previa and Placenta Accrete: Careful Preparation and Critical Management. OGS NOW, No.9. (Japanese). Tokyo: Medical View; 2012:94-99. Copyright @ Medical View).

Pay attention to the presence of the lumbar artery coming out from beneath (-Fig. 2).

- Dissect along the arterial wall at a right angle from the lower right side of the aorta using dissecting forceps and penetrate into the lower left side (-Fig. $\mathbf{3}$ ).

- While subtly opening and closing the tip of the dissecting forceps, proceed along the arterial wall toward the contralateral index finger, and resistance is felt at the tip, confirming the correct direction. Caution should be exercised because the lumbar artery might be involved.

\section{Tips and Warnings}

While temporarily cross-clamping the abdominal aorta, the most important consideration is to avoid venous injury and lumbar artery damage. To achieve this, it is important to dissect along the artery wall, place the index finger at the tip of the dissecting forceps, and verify the direction by which the forceps tip advances using the index finger and resistance of the forceps tip and the presence or absence of the lumbar artery (-Fig. $\mathbf{3}$ ).

Step 4: Preparing clamping the aorta.

- Place a vascular tape on the aorta and lift in a perpendicular manner.

- To prevent clamping and injuring the vein with the crossclamp, slightly lift the artery using a vascular tape.

Step 5: Clamping the aorta.

- Clamp the aorta only using a cross-clamp (-Fig. 4).

- When clamping the aorta and interrupting blood flow in a preventive manner prior to hysterectomy, clamp with the forceps after administering 3,000 to 5,000 units of heparin. ${ }^{1,2}$

- Regarding the duration of cross-clamping, there are few reports on such cases in the field of obstetrics; however, it has been reported that $<40$ to 60 minutes is recommended. ${ }^{3}$

- There are various types of cross-clamps such as those with rubber attached to the blade (-Fig. 5) and those that are flexible so as not to disturb the operative field (Cosgrove Flex Clamp, V. Mueller, Allegiance Healthcare Corp., IL) (-Fig. 6).

Step 6: Declamping the aorta

- Following hysterectomy and completion of hemostasis treatment, slowly remove the cross-clap while observing blood pressure.

- When removing the forceps, do so gradually so as not to cause hypotension, with the help of an anesthesiologist. 


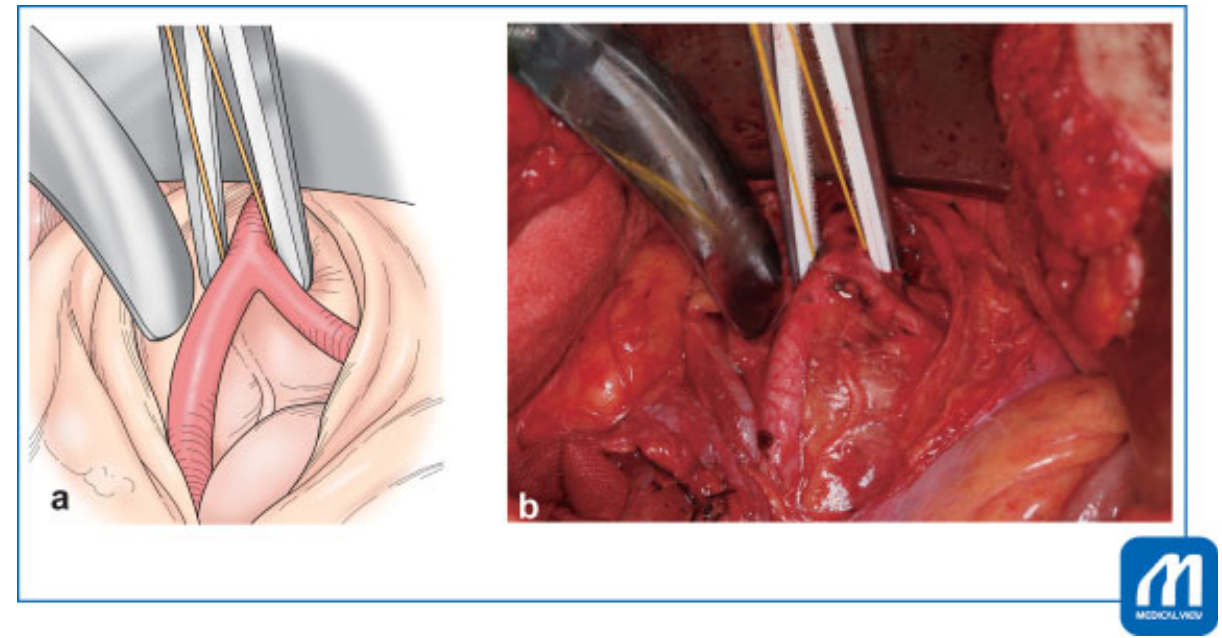

Fig. 4 Clamp the aorta only using a cross clamp forceps while lifting with blood vessel tape. (Reproduced with permission of Hashiguchi M. In: Hiramatsu Y, Konishi I, Sakuragi N, Takeda S, eds. Surgery for Pregnancy with Placenta Previa and Placenta Accrete: Careful Preparation and Critical Management. OGS NOW, No.9. (Japanese). Tokyo: Medical View; 2012:94-99. Copyright @ Medical View).

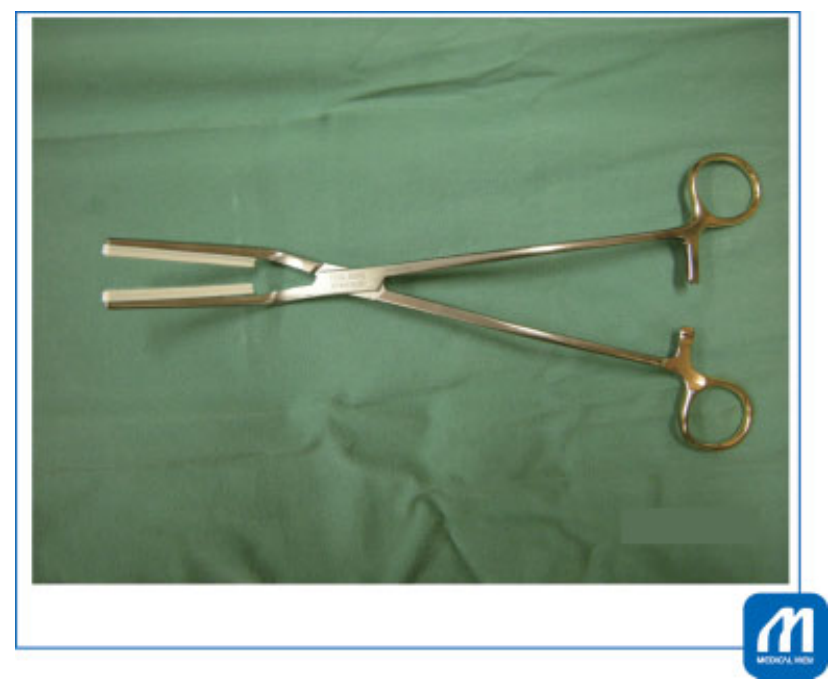

Fig. 5 Common type cross-clamp forceps. (A) lumber artery; (B) Right iliac artery; (C) Left iliac artery. (Reproduced with permission of Hashiguchi M. In: Hiramatsu Y, Konishi I, Sakuragi N, Takeda S, eds. Surgery for Pregnancy with Placenta Previa and Placenta Accrete: Careful Preparation and Critical Management. OGS NOW, No.9. (Japanese). Tokyo: Medical View; 2012:94-99. Copyright @ Medical View).

Confirm the absence of ischemia caused by the formation of thrombus by palpating the pulse of the dorsal arteries of both the feet or by pulse oximeter of the hullax.

- If the surgeon is experienced in lymph node dissection, such as in radical hysterectomy, cross-clamping can be easily learned in several cases under the support of a vascular surgeon and performed in approximately 5 minutes. Moreover, performing cross-clamping when surgical repair is not possible in the event of inferior vena cava injury has no risk; however, it is preferable to perform cross-clamping with the help of a vascular surgeon.

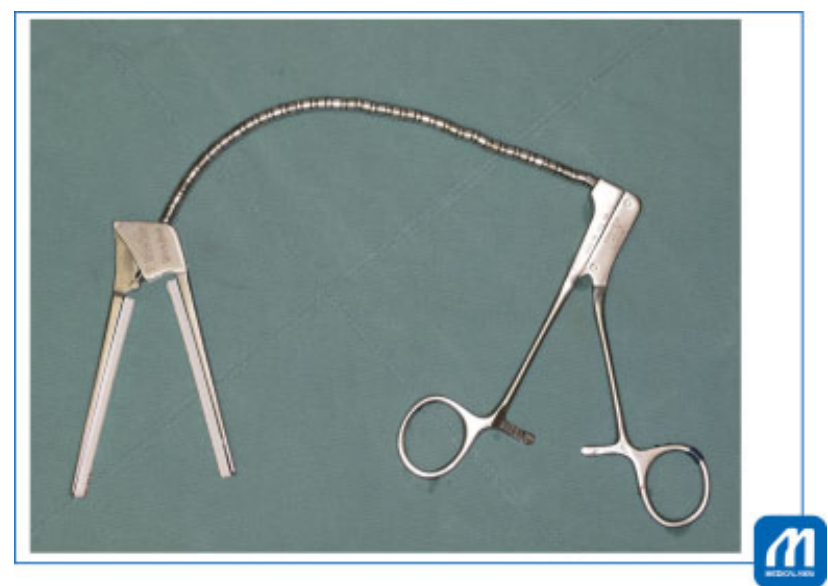

Fig. 6 Cosgrove Flex Clamp, V. Mueller, Allegiance Healthcare Corp., IL. (Reproduced with permission of Hashiguchi M. In: Hiramatsu Y, Konishi I, Sakuragi N, Takeda S, eds. Surgery for Pregnancy with Placenta Previa and Placenta Accrete: Careful Preparation and Critical Management. OGS NOW, No.9. (Japanese). Tokyo: Medical View; 2012:94-99. Copyright (c) Medical View).

\section{Conflict of Interest} None.

\section{Reference}

1 Palacios Jaraquemada JM. Ultrasound interactive case study: conservative treatment in placenta accreta and percreta. Accessed December 3, 2009 at: http://www.obgyn.net/ultrasound/ultrasound.asp?page=/us/present/0107/ jaraquemada

2 Palacios Jaraquemada JM. Aortic cross-clamping in obstetrics. Accessed December 3, 2009 at: http://www.obgyn.net/displayarticleasp? page $=/$ english/pubs/features/POVaortic_cross

3 Chou MM, Ke YM, Wu HC, et al. Temporary cross-clamping of the infrarenal abdominal aorta during cesarean hysterectomy to control operative blood loss in placenta previa increta/percreta. Taiwan J Obstet Gynecol 2010;49(01):72-76 